\title{
Higgs in bosonic channels (CMS)
}

\author{
Valentina Gori ${ }^{1}$ a \\ ${ }^{1}$ Università degli Studi \& INFN Firenze, \\ via G. Sansone, 1 \\ 50019 Sesto Fiorentino (FI) \\ Italy
}

\begin{abstract}
The main Higgs boson decays into bosonic channels will be considered, presenting and discussing results from the latest reprocessing of data collected by the CMS experiment at the LHC, using the full dataset recorded at centre-of-mass energies of 7 and $8 \mathrm{TeV}$. For this purpose, results from the final Run-I papers for the $H \rightarrow Z Z \rightarrow 4 \ell$, $H \rightarrow \gamma \gamma$ and $H \rightarrow W W$ analyses are presented, focusing on the Higgs boson properties, like the mass, the signal strenght, the couplings to fermions and vector bosons, the spin and parity properties. Furthermore, the Higgs boson width measurement exploiting the on-shell versus the off-shell cross section (in the $H \rightarrow Z Z \rightarrow 4 \ell$ and $H \rightarrow Z Z \rightarrow 2 \ell 2 v$ decay channels) will be shown.

All the investigated properties result to be fully consistent with the SM predictions: the signal strength and the signal strength modifiers are consistent with unity in all the bosonic channels considered; the hypothesis of a scalar particle is strongly favored, against the pseudoscalar or the vector/pseudovector or the spin-2 boson hypotheses (all excluded at $99 \% \mathrm{CL}$ or higher in the $H \rightarrow Z Z \rightarrow 4 \ell$ channel).

The Higgs boson mass measurement from the combination of $H \rightarrow Z Z \rightarrow 4 \ell$ and $H \rightarrow \gamma \gamma$ channels gives a value $m_{H}=125.03_{-0.27}^{+0.26}$ (stat. $)_{-0.15}^{+0.13}$ (syst.). An upper limit $\Gamma_{H}<22 \mathrm{MeV}$ can be put on the Higgs boson width thanks to the new indirect method.
\end{abstract}

\section{Introduction}

After the Higgs boson observation in 2012 by the ATLAS and CMS experiments [1, 2], the main goal for both the collaborations is the precise measurement of its properties exploiting the growing statistics, in order to confirm or to find discrepancies with respect to the standard model (SM) predictions for the Higgs properties themselves. We are here presenting the latest results from the CMS collaboration for the main analyses investigating the bosonic decay channels.

\section{Higgs properties measurement in the $H \rightarrow Z Z \rightarrow 4 \ell$ channel}

The properties of a Higgs boson candidate are measured in the $H \rightarrow Z Z \rightarrow 4 \ell$ decay channel, with $\ell=e, \mu$, using data from pp collisions corresponding to an integrated luminosity of $5.1 \mathrm{fb}^{-1}$ at the center-of-mass energy of $\sqrt{s}=7 \mathrm{TeV}$ and $19.7 \mathrm{fb}^{-1}$ at $\sqrt{s}=8 \mathrm{TeV}$, recorded with the CMS detector

\footnotetext{
ae-mail: valentina.gori@cern.ch
} 
at the LHC [3].

This channel has a very low branching ratio $\left(\mathrm{BR} \sim 10^{-4}\right)$; however, it was the "golden" channel for the Higgs discovery, because of its high resolution (only leptons, fully reconstructed final state) and high signal over background ratio. Its sharp mass peak (see Fig. 1) allows for a precise mass measurement. Currently, all measurements are statistically limited.

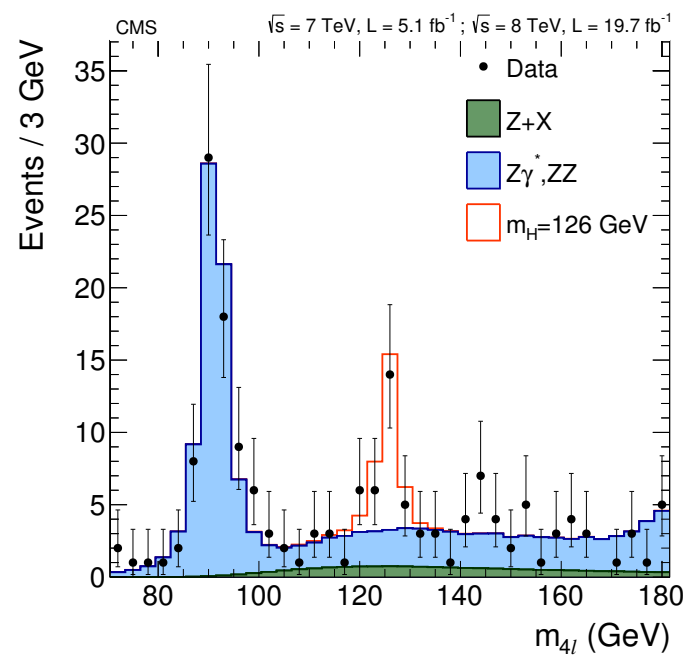

Figure 1. Distribution of the four-lepton reconstructed mass for the sum of the $4 e, 4 \mu$ and $2 e 2 \mu$ channels for the low-mass region. Points with error bars represent the data, shaded histograms represent the backgrounds, and the unshaded histogram the signal expectation for a mass hypothesis of $m_{H}=126 \mathrm{GeV}$. Signal and ZZ background are normalized to the $\mathrm{SM}$ expectation, $\mathrm{Z}+\mathrm{X}$ background to the estimation from data.

The main background sources affecting this channel are given by an irreducible component of nonresonant $Z Z \rightarrow 4 \ell$ production, whose normalization and shape are taken directly from simulated samples, and by a reducible component of $Z+$ jets and $t \bar{t}$ events.

The reconstructed $\mathrm{Z}$ boson masses and five production and decay angles are used to build a kinematic discriminant using matrix elements at Leading Orded to separate signal from background. This technique is known as "MELA" (Matrix Element Likelihood Analysis) and can be also used to build a discriminant aimed at the determination of the spin-parity properties of the resonance, or its width.

The general analysis strategy is to fit the three-dimensional distribution built using the invariant mass of the four leptons in the final state $\left(m_{4 \ell}\right)$, the kinematic discriminant $K D$ built with MELA technique and the transverse momentum of the four leptons system $\left(p_{T}(4 \ell)\right)^{1}$.

The new boson is observed as a narrow resonance with a local significance of 6.8 standard deviations (see Fig. 2a), a measured mass of $125.6 \pm 0.4$ (stat.) \pm 0.2 (syst.) $\mathrm{GeV}$ (see Fig. 2b) and a total width $\leq 3.4 \mathrm{GeV}$ at the $95 \%$ confidence level.

The production cross section of the new boson times its branching fraction to four leptons is measured to be $0.93_{-0.23}^{+0.26}$ (stat.) ${ }_{-0.09}^{+0.13}$ (syst.) times that predicted by the SM (see Fig. 3a).

The signal strength modifiers associated with fermions and vector bosons are compatible with the SM prediction $(1,1)$ as shown in Fig. $3 \mathrm{~b}$.

\footnotetext{
${ }^{1}$ The 3D approach uses $\left(p_{T}(4 \ell)\right)$ for events with no or one reconstructed jet; if two jets are reconstructed, then a Fisher variable is used
} 


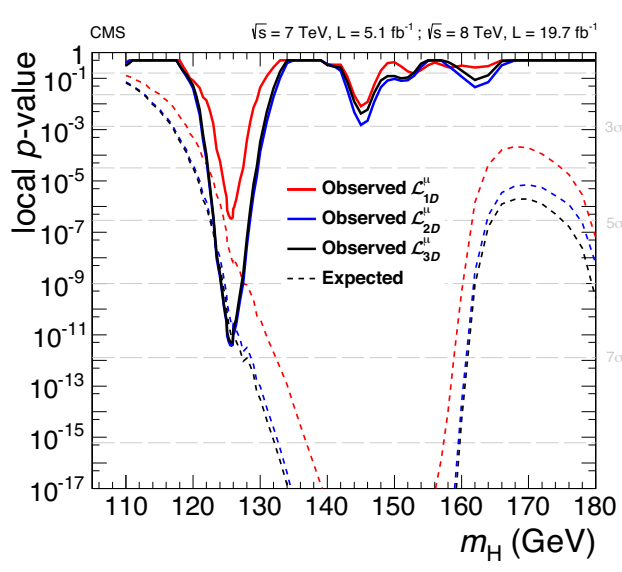

(a)

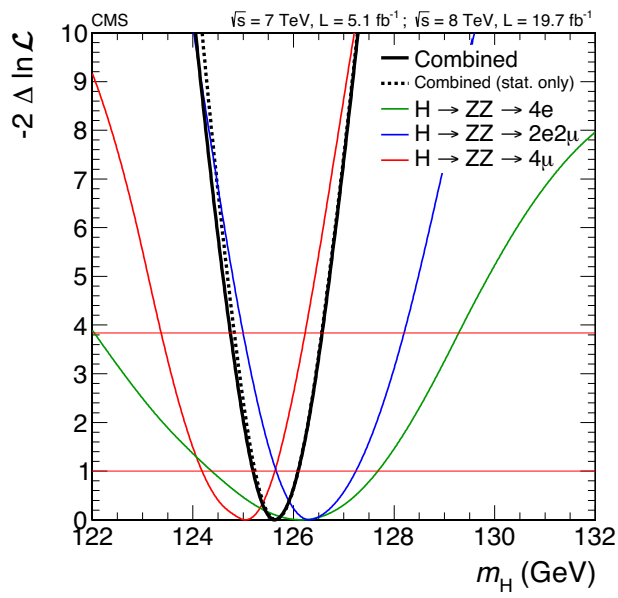

(b)

Figure 2. (a) Significance of the local excess with respect to the SM background expectation as a function of the Higgs boson mass for the 1D fit (using only $m_{4 \ell}$ ), 2D fit (using $m_{4 \ell}$ and $K D$ ), and the nominal 3D fit. Results are shown for the full data sample in the low-mass region only. (b) Scan of the negative log-likelihood versus tested SM Higgs boson mass $m_{H}$ for each of the three channels separately $(4 e, 2 e 2 \mu, 4 \mu)$ and the combination of the three, where dashed line represents the scan including only statistical uncertainties, when using the 3D model.

In this channel, the spin-parity properties of the observed resonance are found to be consistent with the expectations for the SM Higgs boson. The hypotheses of a pseudoscalar and all tested spin-1 boson hypotheses are excluded at the $99 \%$ confidence level or higher (see Fig. 4). All tested spin-2 boson hypotheses are also excluded at the $99 \%$ confidence level or higher.

\section{Width indirect measurement from $H \rightarrow Z Z \rightarrow 4 \ell$ and $H \rightarrow Z Z \rightarrow 2 \ell 2 v$ channels}

Direct measurements of the Higgs boson width performed in the $H \rightarrow Z Z \rightarrow 4 \ell$ decay channel result to be strongly limited by the detector resolution, thus do not allow to give a useful constraint on the resonance width. A tighter constraint on the total Higgs boson width, $\Gamma_{H}$, can be given using both off-shell and on-shell production and decay rates to four leptons, $H \rightarrow Z Z \rightarrow 4 \ell$, or two leptons plus two neutrinos, $H \rightarrow Z Z \rightarrow 2 \ell 2 v$, with $\ell=e, \mu$. The analysis [4] is based on the data collected by the CMS experiment at the LHC in 2011 and 2012, corresponding to integrated luminosities of 5.1 $\mathrm{fb}^{-1}$ at a center-of-mass energy $\sqrt{s}=7 \mathrm{TeV}$ and $19.7 \mathrm{fb}^{-1}$ at $\sqrt{s}=8 \mathrm{TeV}$. The $4 \ell$ analysis uses the $\mathrm{ZZ}$ invariant mass distribution as well as a matrix element likelihood discriminant to separate the $\mathrm{ZZ}$ components originating from gluon- and quark-initiated processes. The $2 \ell 2 v$ analysis relies on the transverse mass or missing transverse energy distributions in jet categories. A simultaneous maximum likelihood fit to the measured kinematic distributions near the resonance peak and above the Z-boson pair production threshold leads to an upper limit on the Higgs boson width of $\Gamma_{H}<22 \mathrm{MeV}$ at a 95\% confidence level (see Fig. 5), which is 5.4 times the expected value in the SM at the measured mass of $m_{H}=125.6 \mathrm{GeV}$.

This result considerably improves over previous experimental constraints from the measurement near the resonance peak. 


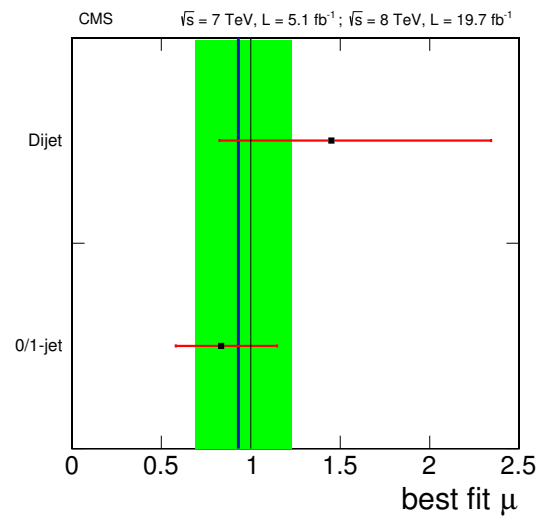

(a)

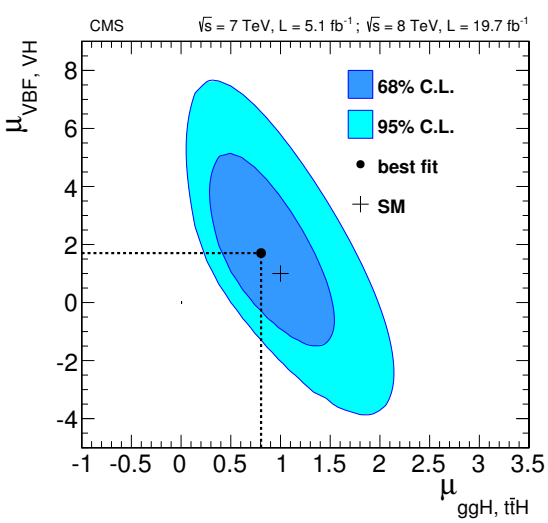

(b)

Figure 3. (a) Values of $\mu$ for dijet and $0 / 1$ jet categories. The vertical line shows the combined $\mu$ together with its associated $\pm 1 \sigma$ uncertainties shown as green band. The horizontal bars indicate the $\pm 1 \sigma$ uncertainties in $\mu$ for the different categories. The uncertainties include both statistical and systematic sources. (b) Likelihood contours on the signal strength modifiers associated with fermions and vector bosons shown at a $68 \%$ and 95\% CL.

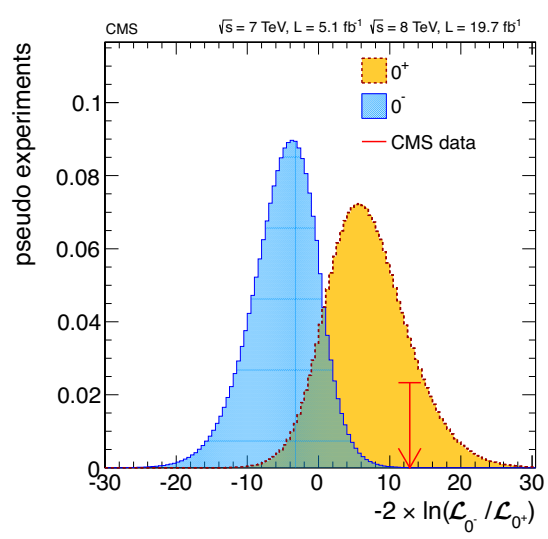

Figure 4. Distribution of a test-statistic $q=-2 \ln \left(\mathcal{L}_{0^{-}} / \mathcal{L}_{0^{+}}\right)$of the pseudoscalar boson hypothesis tested against the SM Higgs boson hypothesis. Distributions for the SM Higgs boson are represented by the yellow histogram and for the alternative $J^{P}$ hypotheses by the blue histogram. The arrow indicates the observed value.

\section{$4 H \rightarrow \gamma \gamma$}

Observation of the diphoton decay mode of the recently discovered Higgs boson and measurement of some of its properties are reported. The analysis [7] uses the entire dataset collected by the CMS experiment in proton-proton collisions during the 2011 and 2012 LHC running periods. The data samples correspond to integrated luminosities of $5.1 \mathrm{fb}^{-1}$ at $\sqrt{s}=7 \mathrm{TeV}$ and $19.7 \mathrm{fb}^{-1}$ at $8 \mathrm{TeV}$. The considered decay channel has a low branching ratio ( $0.2 \%$ at $125 \mathrm{GeV})$. On the other hand, it has a clean final state, which allows high precision for mass reconstruction and measurement (see in Fig. 6 


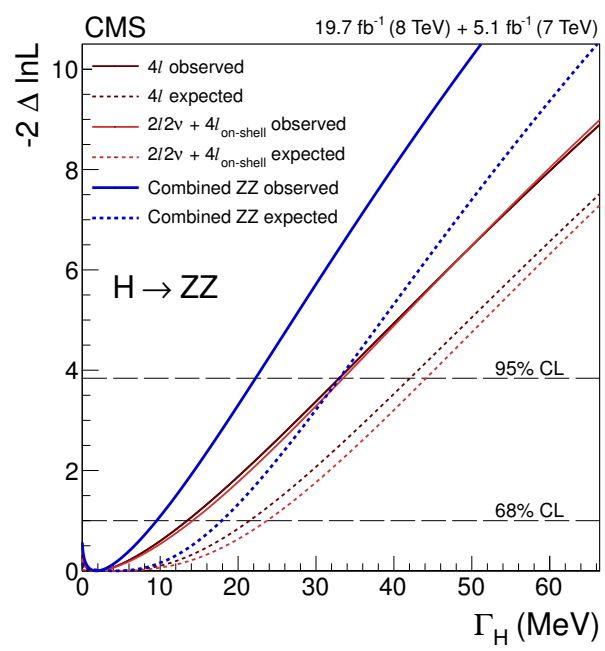

Figure 5. Likelihood scan versus the $\Gamma_{H}$ variable. Different colors refer to: combination of $4 \ell$ low-mass and high-mass (ochre), combination of $4 \ell$ low-mass and $2 \ell 2 v$ high-mass (Sienna) and combination of $4 \ell$ low-mass and both channels at high-mass (blue). Solid lines represent observed limits, while dashed lines are the expected.

the mass spectrum). The analysis is performed using several multivariate classifiers. The new analysis shows considerable enhancements with respect to the previous one [5]:

- an improved intercalibration of electromagnetic calorimeters;

- an improved energy regression algorithm, leading to a better energy resolution which gives a reduction by a factor $\sim 3$ on the systematic uncertainties on the mass measurement;

- a completely re-optimised analysis chain;

- a new method for modeling the background.

The analysis strategy consists in performing a simultaneous fit to the mass distribution $m_{\gamma \gamma}$ in the various event classes. The signal model is derived from Monte Carlo and a $Z \rightarrow e e$ sample is used to estimate the accuracy of the signal simulation. The background is obtained by a fit to data.

The main background sources are an irreducible background given by prompt diphoton production (about $70 \%$ of the total background) and a reducible background given by $\gamma+$ jet events (about $30 \%$ contribution) or dijet events $(<1 \%)$.

Multivariate (MVA) discriminants are used to calculate the photon energy corrections, to find the diphoton vertex location, and to separate prompt photons from misidentifications of jet fragments using shower shape and isolation variables.

Event classification is firstly performed based on the production mechanism: categories are "tagged" by the presence of additional objects in the final state. Remaining untagged events (about $99 \%$ of the total amount) are then classified according to a diphoton multivariate discriminant which is based on kinematics and mass resolution and takes has high values for events with good diphoton mass resolution and high probability of being signal rather than background (see Fig. 7).

A clear signal is observed at a mass close to $125 \mathrm{GeV}$ with a local significance of $5.7 \sigma$, where a significance of $5.2 \sigma$ is expected for the SM Higgs boson (Fig. 8a). 


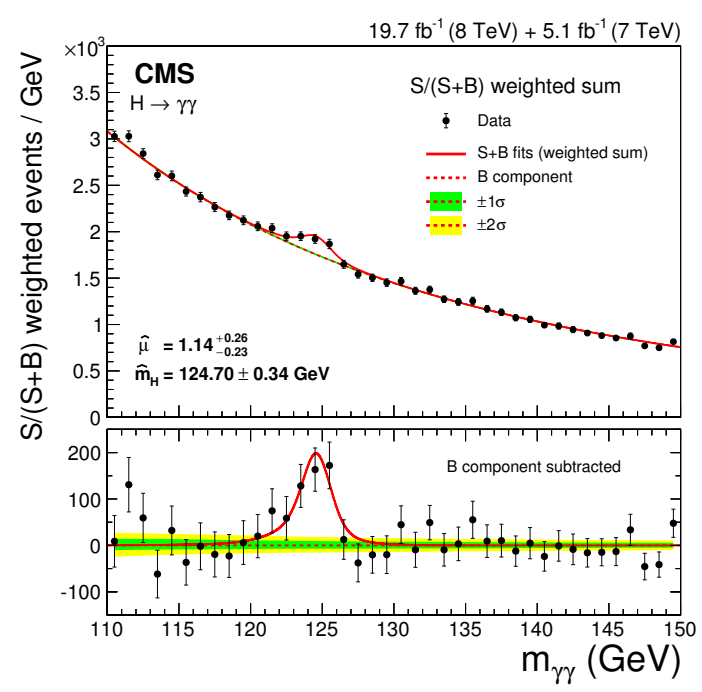

Figure 6. Diphoton mass spectrum weighted by the ratio $S /(S+B)$ in each event class, together with the background subtracted weighted mass spectrum.

The mass is measured to be $124.70 \pm 0.34 \mathrm{GeV}=124.70 \pm 0.31$ (stat.) \pm 0.15 (syst.) GeV (Fig. 8 b). The best-fit signal strength relative to the SM prediction is $1.14_{-0.23}^{+0.26}$ (Fig. 9a); the signal strength modifiers associated with different production mechanisms show compatibility between categories, production modes and with the SM (Fig. 9b).

The Landau-Yang theorem forbids the direct decay of a spin-1 particle into a pair of photons. However, it is of interest to compare the hypothesis of a spin-2 "graviton-like" model with minimal couplings, $2_{m}^{+}$, to that of a spin-0 SM-Higgs-boson-like, $0^{+}$model. The hypothesis of the signal being $2_{m}^{+}$is disfavoured. When produced entirely by gluon fusion, it is disfavoured at 94\% CL.

\section{Mass combined measurement}

A precise value for the Higgs boson mass can be obtained from the combination of $H \rightarrow Z Z \rightarrow 4 \ell$ and $H \rightarrow \gamma \gamma$ channels.

The value reported in [6] is $m_{H}=125.03_{-0.27}^{+0.26}$ (stat. $)_{-0.15}^{+0.13}$ (syst.) $\mathrm{GeV}$, with the precision dominated by the statistical uncertainty.

In Fig. 10a the result for the combination, together with results for the single channels, are shown. Fig. 10b gives an indication of the mass difference between the two channels.

\section{$6 H \rightarrow W W$ with leptonic final states}

A search for the standard model Higgs boson decaying to a W-boson pair at the LHC is reported. The event sample corresponds to an integrated luminosity of $4.9 \mathrm{fb}^{-1}$ and $19.4 \mathrm{fb}^{-1}$ collected with the CMS detector in pp collisions at $\sqrt{s}=7$ and $8 \mathrm{TeV}$, respectively [8]. The Higgs boson candidates are selected in events with two or three charged leptons.

This decay channel has a high branching ratio $(\sim 22 \%)$ for a Higgs boson mass of about $125 \mathrm{GeV}$. 


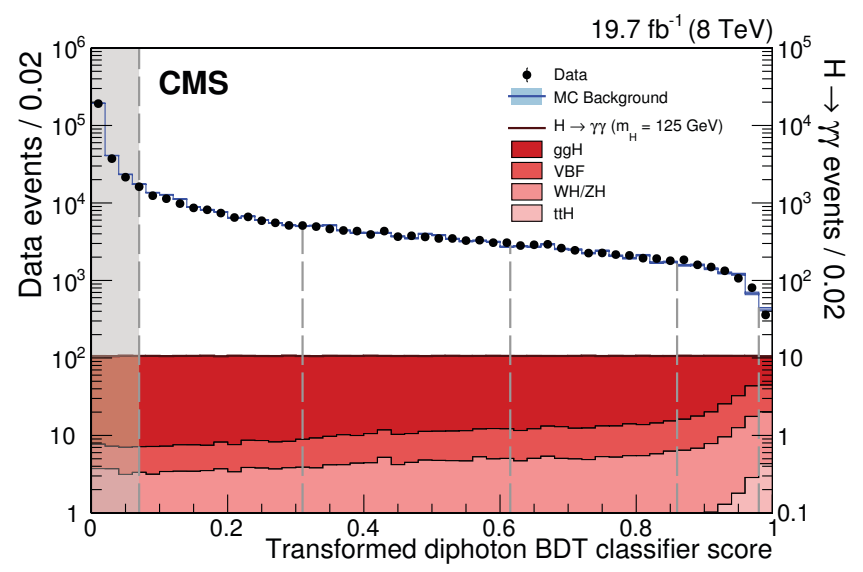

Figure 7. Transformed diphoton BDT classifier score for events satisfying the full diphoton preselection in the $8 \mathrm{TeV}$ data (points with error bars, left axis), and for simulated signal events from the four production processes (solid filled histograms, right axis). The discriminant is transformed such that the sum of signal events has a flat distribution, to assist visualization of the performance of the BDT. The outlined histogram, following the data points, is for simulated background events. The vertical dashed lines show the boundaries of the untagged event classes, with the leftmost dashed line representing the score below which events are discarded and not used in the final analysis. The signal to background ratio increases with the classifier score. Events produced via Vector Boson Fusion as well as associated production to a vector boson or a top-antitop pair achieve high scores, due to their harder $p_{T}^{\gamma \gamma}$ spectrum.

Actually, only the fully leptonic $(e, \mu)$ final states are used here, i.e. about $4.5 \%$ of the total $\mathrm{BR}$, hence about $1 \%$. In this decay channel, the final state is not completely reconstructed because of neutrinos, so a broad signal mass peak is observed.

The analysis is performed requiring two high-quality opposite-sign leptons and missing transverse energy (MET) and exploiting angular correlations to reject background. The main background sources are the non-resonant WW production and $\mathrm{W}+$ jets in the dilepton category, top production in events with high jet multiplicity and non-prompt leptons (i.e. $t \bar{t}$ events) for the tri-lepton categories. Also Drell-Yan $\mathrm{Z} / \gamma^{*}+$ jets is a background source.

Events are categorized according to the production mechanism. In the $0 / 1$ jet gluon fusion category, both a cut\&count analysis and a $2 \mathrm{D}$ shape analysis using the variables $\left(m_{\ell \ell}, m_{T}\right)$ are performed, and about 100 total signal events are expected ${ }^{2}$. In the 2 jets VBF category a template fit to the $m_{\ell \ell}$ distribution is performed; few signal events are expected, with a signal to background ratio of about 1. In the 2 jets VH category a counting analysis is performed; few signal events are expected, with a signal to background ratio of about 0.1 . In the $W H \rightarrow 3 \ell 3 v, Z H \rightarrow 3 \ell v+2$ jets categories, shapebased analyses are performed, with no significant excess of events.

Combining the categories, an excess of events above background is observed, consistent with the expectation from the SM Higgs boson with a mass of around $125 \mathrm{GeV}$. The probability to observe an excess equal or larger than the one seen, under the background-only hypothesis, corresponds to a significance of 4.3 standard deviations for $m_{H}=125.6 \mathrm{GeV}$ (with an expected significance of $5.8 \sigma$ ).

\footnotetext{
${ }^{2}$ Here the $m_{\ell \ell}$ is the dilepton invariant mass and $m_{T}$ is the transverse mass defined as $m_{T}=\sqrt{2 p_{T}^{\ell \ell} E_{T}^{\text {miss }}\left(1-\cos \Delta \Phi_{\ell \ell-E_{T}^{m i s s}}\right)}$, where $E_{T}^{\text {miss }}$ is the missing transverse energy.
} 


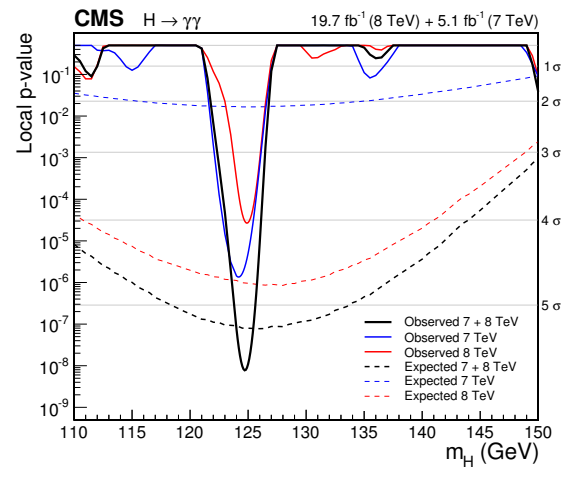

(a)

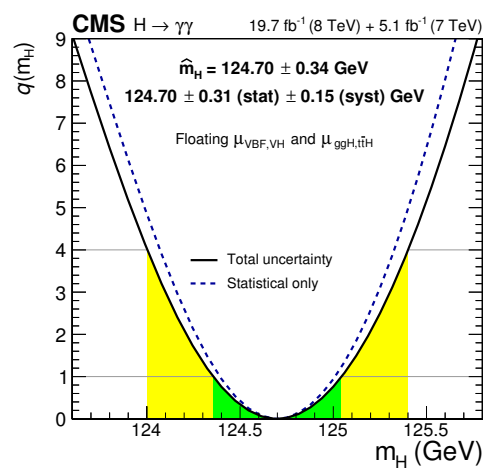

(b)

Figure 8. (a) Local p-values as a function of $m_{H}$ for the $7 \mathrm{TeV}, 8 \mathrm{TeV}$ and the combined dataset. The values of the expected significance, calculated using the background expectation obtained from the signal-plus-background fit, are shown as dashed lines. (b) Scan of the likelihood ratio, $q$, as a function of the hypothesised mass when $\mu_{g g H, t t H}$ and $\mu_{V B F, V H}$ are allowed to vary independently.

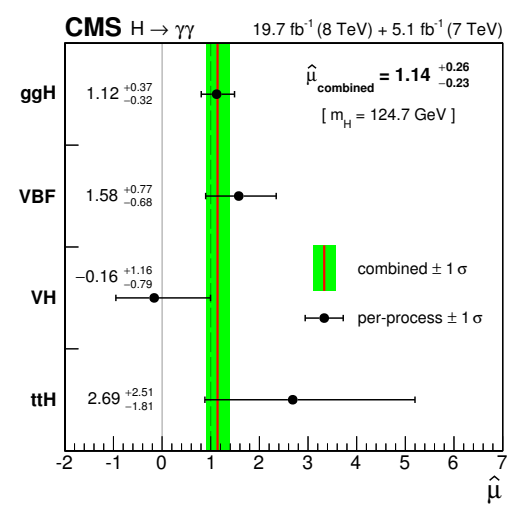

(a)

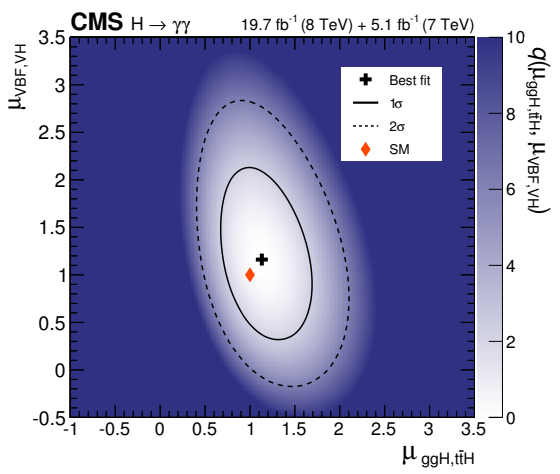

(b)

Figure 9. (a) Best-fit signal strength measured for each of the production processes in a combined fit where the signal strengths of all four processes have been allowed to vary independently in the fit. The horizontal bars indicate $\pm 1 \sigma$ uncertainties in the values for the individual processes. The green band corresponds to $\pm 1 \sigma$ uncertainties in the value obtained from the combined fit with a single signal strength. (b) Map of the likelihood ratio $q\left(\mu_{q q H, t t H}, \mu_{V B F, V H}\right)$ with $m_{H}$ treated as a nuisance parameter. The $1 \sigma$ and $2 \sigma$ uncertainty contours are shown. The cross indicates the best-fit values $(1.13,1.15)$, and the diamond represents the SM expectation.

The observed signal cross section times the branching fraction to $\mathrm{WW}$ for $m_{H}=125.6 \mathrm{GeV}$ is $0.72_{-0.18}^{+0.20}$ times the SM expectation (Fig. 11a).

The likelihood profiles on the signal strength modifiers $\mu_{g g H}$ and $\mu_{V B F, V H}$ are shown in Fig. 11b.

The spin-parity $J^{P}=0^{+}$hypothesis is favored against a narrow resonance with $J^{P}=2^{+}$or $J^{P}=0^{-}$ decaying to a W-boson pair. This result provides strong evidence for a Higgs-like boson decaying to a W-boson pair. 


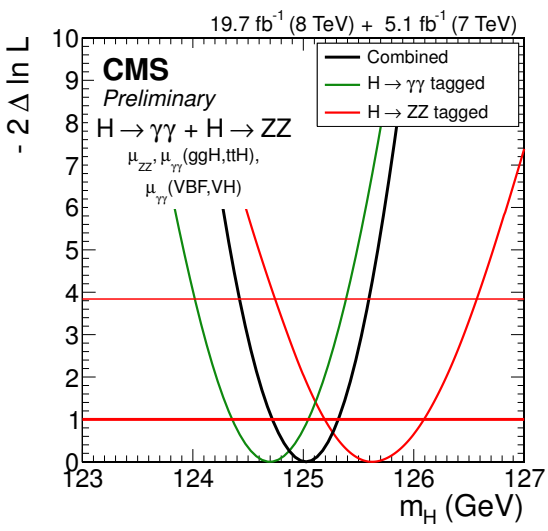

(a)

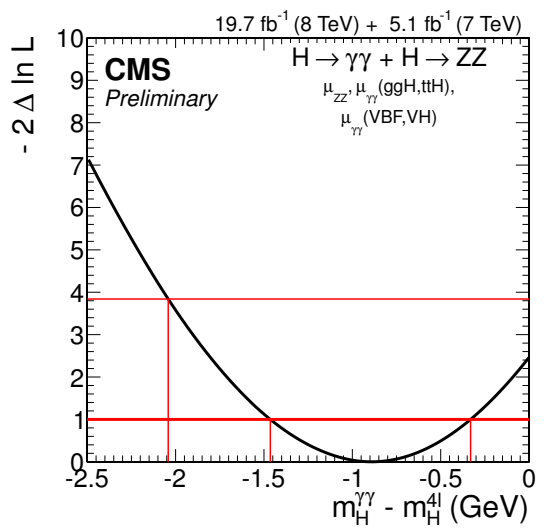

(b)

Figure 10. (a) 1D test statistics $q\left(m_{H}\right)$ scan versus the hypothesized Higgs boson mass $m_{H}$ for the $\gamma \gamma$ (green) and $4 \ell$ (red) final states separately and for their combination (black). (b) $1 \mathrm{D}$ test statistics $q\left(m_{H}^{\gamma \gamma}-m_{H}^{4 \ell}\right)$ scan vs the difference between two individual mass measurements from $\gamma \gamma$ and $4 \ell$ final states. The crossings with the thick (thin) horizontal lines define the 68\% (95\%) CL interval for the measured difference.

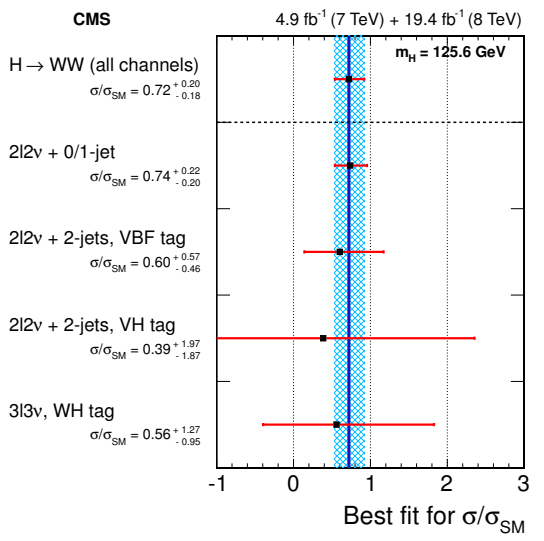

(a)

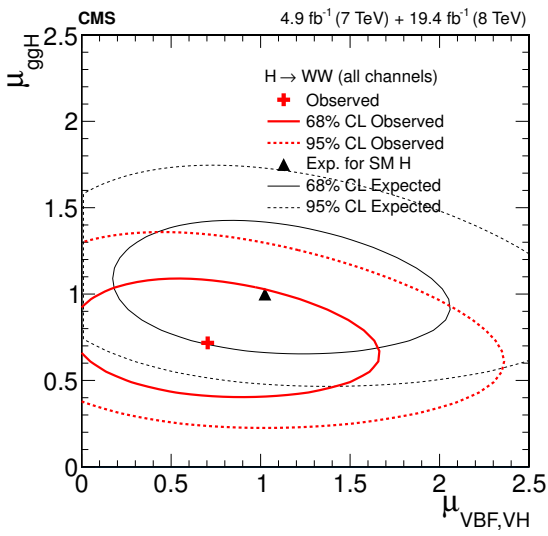

(b)

Figure 11. (a) Observed $\sigma / \sigma_{S M}$ for $m_{H}=125.6 \mathrm{GeV}$ for each category used in the combination. The observed $\sigma / \sigma_{S M}$ value in the $\mathrm{ZH}$ to $3 \ell v+2$ jets category is $6.41_{-6.38}^{+7.43}$. Given its relatively large uncertainty with respect to the other categories it is not shown individually, but it is used in the combination. (b) Likelihood profiles on $\mu_{g g H}$ and $\mu_{V B F, V H}$ at $68 \%$ (solid) and 95\% CL (dotted). The expected (black) and observed (red) distributions for $m_{H}=125.6 \mathrm{GeV}$ are shown.

\section{Conclusions}

The analysis of Higgs boson decays in bosonic channels has been crucial for the Higgs boson discovery and is now important for mass and properties determination. A precise mass measurement can be obtained from the combination of $H \rightarrow Z Z \rightarrow 4 \ell$ and $H \rightarrow \gamma \gamma$ channels; a constraint on the Higgs boson width can be derived from off-peak and on-peak cross sections measurements exploiting 
$H \rightarrow Z Z$ decays with final states in $4 \ell$ and $2 \ell 2 v$. Spin/parity studies are all confirming the scalar hypothesis. The signal strengths and couplings are also consistent with the SM hypothesis.

\section{References}

[1] ATLAS Collaboration, G. Aad et al., "Observation of a new particle in the search for the Standard Model Higgs boson with the ATLAS detector at the LHC", Phys.Lett. B 716 1-29 (2012).

[2] CMS Collaboration, S. Chatrchyan et al., "Observation of a new boson at a mass of $125 \mathrm{GeV}$ with the CMS experiment at the LHC", Phys.Lett. B 716 30-61 (2012).

[3] CMS Collaboration, S. Chatrchyan et al., "Measurement of the properties of a Higgs boson in the four-lepton final state", Phys.Rev. D 89092007 (2014).

[4] CMS Collaboration, S. Chatrchyan et al., "Constraints on the Higgs boson width from off-shell production and decay to Z-boson pairs", Phys.Lett. B 73664 (2014).

[5] CMS Collaboration, S. Chatrchyan et al., "Search for the standard model Higgs boson decaying into two photons in $p p$ collisions at $\sqrt{s}=7$ TeV", Phys. Lett. B 710403 (2012).

[6] CMS Collaboration, S. Chatrchyan et al., "Precise determination of the mass of the Higgs boson and studies of the compatibility of its couplings with the standard model", CMS-PAS-HIG-14-009.

[7] CMS Collaboration, S. Chatrchyan et al., "Observation of the diphoton decay of the Higgs boson and measurement of its properties", arXiv:1407.0558 [hep-ex].

[8] CMS Collaboration, S. Chatrchyan et al., "Measurement of Higgs boson production and properties in the WW decay channel with leptonic final states", JHEP01 096 (2014). 